\title{
Verfügbarkeit intralogistischer Systeme
}

\author{
DIPL.-ING. GUIDO FOLLERT \\ DIPL.-ING. LARS NAGEL \\ LEHRSTUHL FÜR FÖRDER- UND LAGERWESEN, UNIVERSITÄT DORTMUND
}

\section{Zusammenfassung}

Bei der Entwicklung und Auslegung komplexer Materialflusssysteme bilden heutzutage die Erreichung einer hohen Leistungsfähigkeit bei vergleichsweise niedrigen Kosten und einem hohen Servicegrad die maßgeblichen Zielkriterien. In zweiter Reihe eng damit verbunden ist der Kennwert Verfügbarkeit, der das Verhältnis zwischen der Ausfall- und der theoretischen Nutzungszeit dieser Systeme beschreibt. Der Kennwert Verfügbarkeit wird für technische Systeme allgemein in einer Vielzahl unterschiedlicher Richtlinien und Normen definiert. Einige davon sind speziell auf den Bereich der Materialflusssysteme ausgerichtet und dokumentieren auch Berechnungsansätze für die Verfügbarkeit. Trotz dieser Anleitungen ist die Analyse und Bewertung von Verfügbarkeitskennwerten bei der Entwicklung und Inbetriebnahme von Materialflusssystemen wiederholt Gegenstand von Auseinandersetzungen zwischen Kunden und Lieferanten. Der vorliegende Beitrag analysiert diese Situation und skizziert Lösungsansätze.

\begin{abstract}
Nowadays complex material flow systems are mostly developed and designed with regard to high performance in line with relatively low costs and a high service level. The second important, closely related parameter is the availability which describes the relation between the breakdown and theoretical operating times of these systems. With regard to technical systems availability is defined by a variety of regulations and standards some of which specially refer to material flow systems and document availability assessments. Despite these guidelines the analysis and evaluation of availability measures during the development and implementation of material flow systems often lead to conflicts between customer and supplier. Such a situation is outlined and studied in this paper.
\end{abstract}

\section{Einleitung}

Die Spanne von den Elementen und Triebwerken der Fördertechnik über komplexe intralogistische Systeme mit umfangreicher Automatisierung bis in die nahe Zukunft mit weitgehender Selbststeuerung der Transportobjekte kennzeichnet die Innovationsfähigkeit der Unternehmen, die sich mit Materialflusstechnik und -steuerung auseinandersetzen. In den zurückliegenden 4 Jahrzehnten wurde auf den Entwicklungen mechanischer Elemente und Maschinen aufbauend mit Hilfe umfassenderer Steuerungen langfristig auf die aktuelle Form intralogistischer Systeme hingearbeitet. Heutzutage bilden vernetzte Maschinen mit leistungsfähiger Steuerungstechnik, die durch Informations- und Kommunikationstechnologien in ihre Umgebung eingebettet sind, integrierte intralogistische Systeme, die sich durch hohe Komplexität auszeichnen können.

Dabei bilden die Routen- und Reihenfolgeprobleme mit dem verbundenen Rechenaufwand für die optimierte Organisation der Abläufe die besonderen Kennzeichen der systemimmanenten Komplexität. Aufbau und Anwendung von Routingtabellen und -strategien gehören gleichermaßen in diese Kategorie wie Algorithmen zur Batchbildung und zur Auftragsauswahl. Darüber hinaus wird durch die Einbettung in ein dynamisches Umfeld die zunehmende Varietät eines Systems charakterisiert. Über wechselnde Last- und Störgrößen an den Schnittstellen wird das erwartete Verhalten des Systems beeinflusst. Hierzu gehören variierende Ausprägungen der Auftragslasten genau so wie wechselnde Zeitabstände für die Vorgänge an den Schnittstellen.

Prototypische Beispiele für komplexe intralogistische Systeme lassen sich in vielen verschiedenen Anwendungsgebieten der Materialflusstechnik identifizieren. Dazu gehören große Gepäckförderanlagen an Flughäfen, die Paketumschlagszentren im Transportgewerbe oder Distributionszentren im Handel mit der umfangreichen Kommissionierung aus einem heterogenen Warensortiment. Insbesondere bei den zuvor genannten, aber auch bei kleineren Systemen mit weniger komplizierten Strukturen, zeigt sich der Einfluss des Kenwertes Verfügbarkeit, der im vorliegenden Beitrag in Bezug auf seine Aussagekraft untersucht wird. Die Verfügbarkeit beschreibt dabei das Verhältnis zwischen der Ausfall- und der theoretischen Nutzungszeit eines 
Systems, berücksichtigt aber auch die Ursache von Störungen, die zur Unterbrechung des Betriebes geführt haben.

Der Vergleich der beispielhaften genannten intralogistischen Systeme zeigt, dass sich diese nicht durch eine singuläre Leistungskenngrößen umfassend beschreiben lassen. Vielmehr spiegeln sich Komplexität und Varietät dieser Systeme in der Weise wider, dass eine weitgehende Charakterisierung der Leistungsfähigkeit nur durch sich ergänzende Leistungskenngrößen möglich ist. Dies wird daran deutlich, dass der Kennwert Durchsatz allein nicht ausreichend ist, um die Leistung eines der Systeme auszudrücken. Ergänzende Informationen zu Durchlaufzeiten sowie Kommissionier-, Sortier-, Transport- und Ein-/Auslagerleistung gestatten erst eine weitergehende Festlegung der Systemeigenschaften.

Die vorliegende Untersuchung wird dadurch erforderlich, dass der Kennwert Verfügbarkeit der zuvor skizzierten Charakterisierung eines Systems durch verschiedene Leistungskenngrößen nicht mehr gerecht wird. Bedingt durch die Komplexität und Varietät ist die Aussagekraft des hoch verdichteten Kennwertes bei der Verwendung für große und vernetzte Systeme anzuzweifeln. In diesem Zusammenhang sollen außerdem die verschiedenen Motivationen und Intentionen der Beteiligten einer Untersuchung von Systemverfügbarkeiten analysiert werden. Ergänzt wird dies durch einen Ausblick auf die Berechnungsansätze für die Verfügbarkeit.

Im Weiteren müssen die Umgebungsbedingungen für intralogistische Systeme, die permanenten Veränderungen unterlegen sind, für die Verfügbarkeitsberechnung Berücksichtigung finden. Diese Dynamik der Umgebung findet ihre Reaktion in zunehmenden Anforderungen an die Flexibilität von Materialflusssystemen [Günt02 S. 8; Schm05, S. 1]. Die Dynamik lässt sich einordnen in den in Abbildung 1 dargestellten Nutzungszyklus für intralogistische Systeme, der sich ausgehend von einem Planungsinitial in einen Planungs- und Realisierungszyklus erstreckt. Dieser Zyklus wird geschlossen durch eine Analysephase, die das Initial für weitere Umplanungen mit anschließender Realisierung bilden. Die ansteigende Häufigkeit der Umplanungen wird induziert durch die Dynamik der Netzwerke, in die intralogistische Systeme eingebettet sind. Die reduzierte Betrachtung des Nutzungszyklus dient im Folgenden zur Referenzierung der unterschiedlichen Einsatzmöglichkeiten einer erweiterten Verfügbarkeitsbetrachtung.

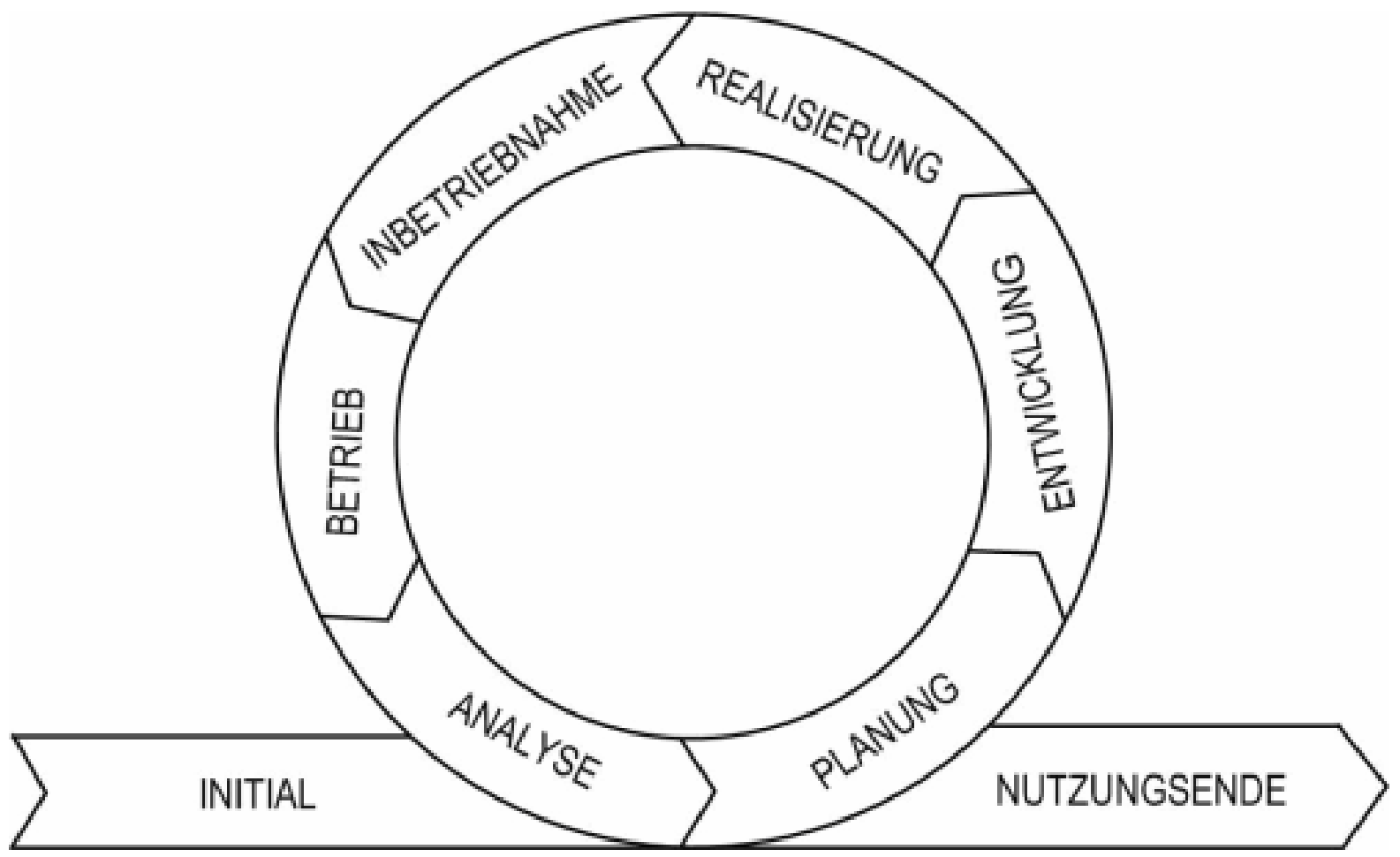

Abbildung 1: Lebenszyklus eines intralogistischen Systems

Im Anschluss an die folgende Betrachtung der verfügbaren Richtlinien für die Untersuchung der Verfügbarkeit von Materialflusssystemen stehen die Analysen für Motivation, Ablauf und Vorgehensweise bei der Verfügbarkeitsberechnung im Blickpunkt. Die dabei identifizierten Schwachstellen dienen als Eckpunkte für die Entwicklung von Lösungsansätzen. Darüber hinaus existierende Kohärenzen mit parallel verwendeten Methoden, wie beispielsweise der Materialflusssimulation mit ihrem Fokus in den Bereichen Leistungsermittlung und Strategieentwicklung, finden ebenfalls Berücksichtigung. 


\section{Aktuelle Richtlinien und verwendete Methoden}

Im Zusammenhang mit der Charakterisierung des Störverhaltens eines Materialflusssystems werden in der Praxis häufig die Begriffe „Zuverlässigkeit“ und „Verfügbarkeit“ synonym verwendet, obgleich sich die beiden Kenngrößen grundlegend unterscheiden.

Die Zuverlässigkeit beschreibt ein System oder ein Systemelement lediglich bis zu seinem ersten Ausfall und gibt die Wahrscheinlichkeit für das Überleben der Einheit in einem bestimmten Zeitraum an. In der Literatur wird die Zuverlässigkeit, die häufig auch mit Funktionszuverlässigkeit bezeichnet wird, definiert als „die Wahrscheinlichkeit dafür, dass eine Betrachtungseinheit während einer definierten Zeitdauer unter angegebenen Funktions- und Umgebungsbedingungen nicht ausfällt" [VDI 4001-2, S. 24]. Unter dem Begriff „Betrachtungseinheit“ oder kurz „Einheit“ kann ein System, ein Teilsystem oder ein Systemelement verstanden werden. Diese Definition gilt vor allem für kontinuierlich belastete Einheiten, die über lange Zeiträume betrachtet werden. Für Materialflusssysteme, deren Teilsysteme und Elemente in der Regel diskontinuierlichen Belastungen unterliegen, wird die Zuverlässigkeit oftmals ereignisbezogen definiert als „die Wahrscheinlichkeit, eine betrachtete Funktion unter angegebenen Randbedingungen störungsfrei und korrekt auszuführen“ [VDI 3581, S. 2]. Zusammenfassend kann festgestellt werden, dass die Kenngröße „Zuverlässigkeit“ lediglich eine Auskunft über die Ausfallwahrscheinlichkeit und Störanfälligkeit einer Betrachtungseinheit gibt und keine Aussage über die Eigenschaft eines Systems im Störungsfall trifft [FEM 9.222, S. 2].

Die Verfügbarkeit hingegen betrachtet auch den Einfluss der Ausfallzeit nach dem Auftreten einer Störung und berücksichtigt damit sowohl das Ausfall- als auch das Reparaturverhalten eines Systems [VDI 3581, S. 2]. Sie gibt die Wahrscheinlichkeit dafür an, dass sich ein System in einem intakten Zustand befindet, und charakterisiert damit die Einsatzbereitschaft eines reparierbaren Systems. Definiert ist die Verfügbarkeit als „die Wahrscheinlichkeit, ein Element oder ein System zu einem vorgegebenen Zeitpunkt in einem funktionsfähigen Zustand anzutreffen“ [VDI 3649, S. 2], [FEM 9.222, S. 3].

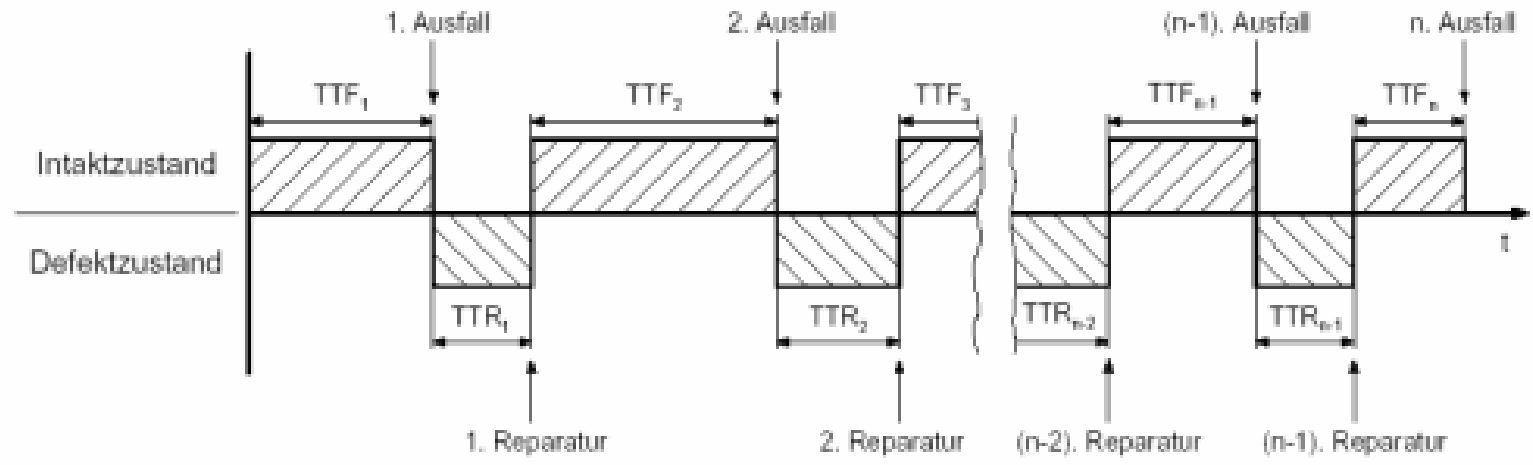

Abbildung 2: Alternierendes Zustandsverhalten eines reparierbaren Systems

Bei Materialflusssystemen handelt es sich um reparierbare Systeme, die während ihrer Einsatzzeit wiederholt zwischen dem Intaktzustand, der durch die Funktionsfähigkeit gekennzeichnet ist, und dem Defektzustand, der eine Störung der Betrachtungseinheit darstellt, wechseln [Gaed77, S. 12]. Der Übergang vom Intakt- in den Defektzustand wird mit „Ausfall“ bezeichnet, während im umgekehrten Fall beim Übergang vom Defekt- in den Intaktzustand eine „Reparatur“ vorliegt. Die Zeitanteile, in denen sich die Betrachtungseinheit im Intaktzustand befindet, werden mit „TTF“ (engl. time to failure - Zeit bis zum nächsten Ausfall; ausfallfreie Zeit) bezeichnet. Die Defektzeitanteile werden mit „TTR“ (engl. time to repair - Zeit für die Reparatur) benannt. Die Wahrscheinlichkeit dafür, dass eine Einheit innerhalb einer bestimmten Zeitdauer den Defektzustand verlässt und wieder funktionsfähig ist, wird durch die Reparaturverteilung abgebildet [Knez93, S. 99].

Die Verfügbarkeit eines Materialflusssystems unterliegt unterschiedlichen Einflussgrößen, wobei die Voraussetzungen für das Erreichen einer hohen Systemverfügbarkeit bereits maßgeblich in der Planungsphase gelegt werden. Hier können Maßnahmen eingeleitet werden, die die spätere Verfügbarkeit des Systems entscheidend beeinflussen [Arno05, S. 298]. Deshalb ist es notwendig, die zu erwartende Verfügbarkeit bereits frühzeitig zu prognostizieren und das Verfügbarkeitsverhalten unterschiedlicher Systemvarianten in der Planungsphase zu untersuchen und zu bewerten. Die bekannten Methoden, die sich mit der Verfügbarkeit beschäftigen, können dies nur an vorhandenen Systemen. Die Prognose ist nicht möglich. 
Bei der Betrachtung von Materialflusssystemen im Zusammenhang mit Zuverlässigkeits- und Verfügbarkeitsbetrachtungen ist der Begriff der „Redundanz“ von hoher Bedeutung. Mit Redundanz wird „das Vorhandensein von mehr funktionsfähigen Mitteln in einer Betrachtungseinheit, als für die Erfüllung der geforderten Funktion notwendig sind“ bezeichnet [Biro91, S. 292]. Redundanz entsteht durch das Einführen zusätzlicher Reserveelemente, welche dieselbe Funktion wie andere Elemente erfüllen, und führt dadurch zu einer Erhöhung der Verfügbarkeit und zur Reduzierung der Ausfallrate einer Betrachtungseinheit.

Zur Berechnung und Verknüpfung der in Zusammenhang mit dem Begriff Verfügbarkeitsprognose genannten Werte kann eine Vielzahl an Verfahren angewendet werden, um unterschiedliche Eigenschaften eines Systems hinsichtlich seiner Verfügbarkeit optimal bewerten zu können. Bei der Ermittlung der Verfügbarkeit eines komplexen Systems erfolgt die Betrachtung ausgehend von der Systemkomponente hin zum Gesamtsystem. Es wird zunächst für die einzelne Einheit das alternierende Zustandsverhalten untersucht und das Verhältnis von Intaktzeiten zu der Summe aus Intakt- und Defektzeiten zur Bestimmung ihrer Verfügbarkeit gebildet [VDI 4003-1, S. 3]. Aus der Kombination des jeweiligen Zustandsverhaltens der Einzelkomponenten gemäß der Aufbaustruktur des Systems wird die Verfügbarkeit des Gesamtsystems ermittelt. Die Verknüpfung der einzelnen Komponenten erfolgt nach Methoden der Statistik und Wahrscheinlichkeitstheorie. Dabei ist es von entscheidender Bedeutung, wie die einzelnen Komponenten miteinander verknüpft sind. Je nach Anordnung der Komponenten ergibt sich für die Systemverfügbarkeit ein Wert, der deutlich höher oder niedriger als die entsprechenden Einzelwerte liegen kann.

Nach dem bisherigen Stand der Wissenschaft wird in den existierenden Richtlinien bei der Bestimmung der Verfügbarkeit von Materialflusssystemen vornehmlich die Zuverlässigkeitstheorie herangezogen. Im deutschsprachigen Raum existiert eine Reihe von Richtlinien zur Zuverlässigkeitstheorie, die im VDI-Handbuch „technische Zuverlässigkeit“ zusammengefasst sind. Dieses Handbuch beinhaltet insgesamt 62 Richtlinien, die sich in allgemeiner Weise unter anderem mit den Kenngrößen Zuverlässigkeit, Verfügbarkeit und Instandhaltbarkeit, mit statistischen Grundlagen sowie probabilistischen Verfahren zur Systemanalyse beschäftigen. Da es sich bei Materialflusssystemen um reparierbare Systeme handelt, können die Ansätze der Zuverlässigkeitstheorie jedoch nur bedingt übertragen werden und bedürfen daher der Adaption. 


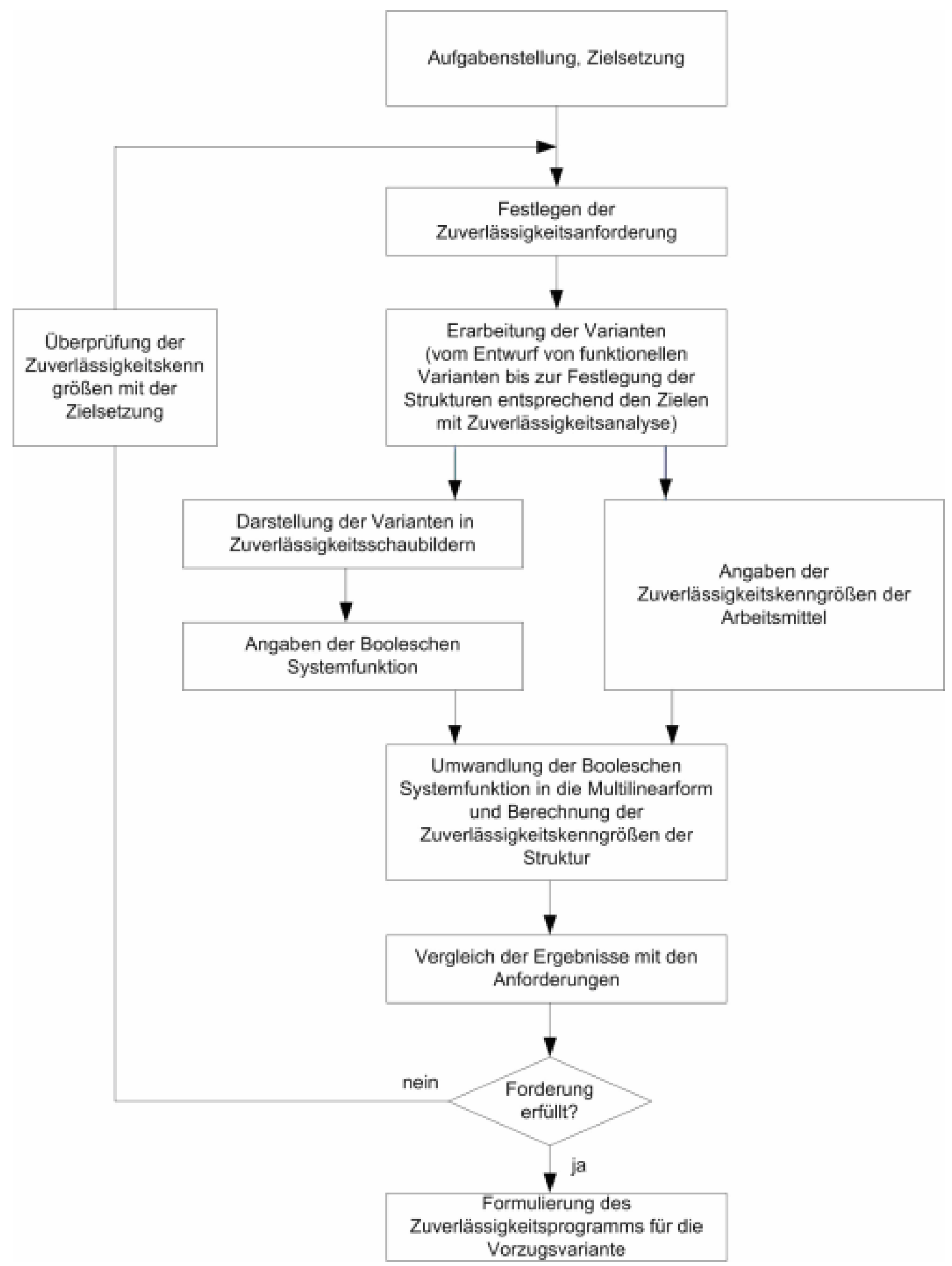

Abbildung 3: Vorgehensweise zur Festlegung eines Verfügbarkeitsprogramms

Neben diesen allgemeinen Richtlinien gibt es nur wenige Regelwerke, die ausdrücklich die Verfügbarkeit von Materialflusssystemen betrachten. Lediglich die FEM-Richtlinien 9.221 und 9.222 sowie die VDI-Richtlinien 3581 und 3649, die nicht Bestandteil des o. g. Handbuchs sind, beschäftigen sich mit diesem Thema. Der Schwerpunkt dieser Richtlinien liegt jedoch im Bereich der Verfügbarkeitstests und Nachweise für bereits realisierte Materialflusssysteme, die sich in der Inbetriebnahmephase befinden. Dabei werden Angaben zum Aufbau und Inhalt von Störprotokollen gemacht, sowie Hinweise zur Auswertung der Testprotokolle und Ermittlung der Verfügbarkeit gegeben. 
Mit den in den Richtlinien beschriebenen Ansätzen zur Prognose der Verfügbarkeit in der Planungsphase lassen sich nur einfache Strukturen abbilden. Dabei wird in einfacher Weise der Einfluss der strukturellen Anordnung der Komponenten auf die Systemverfügbarkeit berücksichtigt [VDI 3649, S. 3-9]. Den Schwerpunkt stellt vor allem die Verfügbarkeitsbestimmung für serielle und parallele Anordnungen nach der Parts-Count-Methode dar. Die Darstellung von relevanten materialflusstechnischen Strukturen wie Redundanzen, Puffern und Brückenanordnungen, sowie zahlreiche wesentliche Einflüsse hinsichtlich des Ausfall- und Reparaturverhaltens oder der Betriebsbedingungen materialflusstechnischer Komponenten werden nur oberflächlich oder gar nicht berücksichtigt. Darüber hinaus ist festzustellen, dass die Richtlinien, die sich explizit mit der Verfügbarkeit von Materialflusssystemen beschäftigen, entscheidende Fehler bei der formalen Modellierung relevanter praktischer Sachverhalte beinhalten [Fran93, S. 303] und Inkonsistenzen bei der einheitlichen Systembeschreibung sowie den anschließenden Aussagen über die Verfügbarkeit des Gesamtsystems aufweisen [Salz87, S. 41]. Als Beispiel dafür ist die Darstellung von Puffern anzuführen, die gemäß den Richtlinien stets über ihren maximalen Füllstand verfügen. Darüber hinaus bleiben zahlreiche Einflüsse und Charakteristika, die bei komplexen Förderund Lagersystemen von wesentlicher Bedeutung sind, unberücksichtigt [Hörs94, S. 22]. Demzufolge können mit Hilfe der bestehenden Prognosemodelle keine qualifizierten Aussagen hinsichtlich des Verfügbarkeitsverhaltens komplexer Materialflusssysteme gemacht werden.

\section{Analyse der Anwendung des Verfügbarkeitskennwertes}

Die Berechnung der Verfügbarkeit eines Materialflusssystems mit den verbundenen Aufwendungen für die Datenerhebung und Auswertung entspringt keinem Selbstzweck. Vielmehr entsteht eine grundlegende Motivation zur Festlegung des Kennwertes durch die funktionalen und wirtschaftlichen Interessen der beteiligten Parteien. Daraus folgen unterschiedliche Intentionen bei der Festlegung von Verfügbarkeitskennwerten im Vertragsverhältnis. Die folgenden Ausführungen basieren auf der Annahme, dass die Auseinandersetzung mit der Verfügbarkeit im Rahmen der Inbetriebnahme eines neuen Materialflusssystems geschieht. Weitergehende Überlegungen zur Verwendung der Verfügbarkeit finden ihren Platz zum Ende dieses Kapitels.

Auf der Seite des Auftraggebers für ein betrachtetes Materialflusssystem dominiert ein Interesse in Bezug auf die Funktionserfüllung dieses Systems. Dessen Auslegung orientiert sich dabei maßgeblich an den planerischen Vorgaben für Durchsatz, Kapazität oder weitere Leistungskenngrößen, wie sie für die beabsichtigte Verwendung des Systems erforderlich sind. Ggf. sind in diesen Kennwerten bereits Aufschläge enthalten, um Leistungsreserven für zukünftige Steigerungen der Anforderungen oder kurzfristige Lastspitzen vorzuhalten. Es muss dem Auftraggeber und zukünftigen Nutzer eines Materialflusssystems aber deutlich sein, dass es sich hierbei um ein störungsbehaftetes technisches System mit systemabhängigen individuellen Eigenschaften handelt. Dieser Sachverhalt, die Ausprägungen der Störungen und die Verantwortung für einzelne Störereignisse werden im Folgenden noch detaillierter betrachtet. Die Tatsache der Störanfälligkeit motiviert einen Auftraggeber aber grundsätzlich dazu, basierend auf dem Verständnis der Leistungseinschränkung durch auftretende Fehler, einen Kennwert für diese Störungen verbindlich einzufordern. Seine Intention ist dabei aber in der Regel die Einschränkung der Leistungsfähigkeit seines Systems in der Messung zu quantifizieren und in der Ausprägung zu minimieren, so dass reziprok hierzu die Verfügbarkeit maximiert wird.

Grundsätzlich folgt ein Hersteller derselben Motivation für die Auseinandersetzung mit der Verfügbarkeit. Er möchte damit aber im positiven Sinne die Störstabilität seiner Anlage dokumentieren. Er sieht den Kennwert in dieser Situation als unabhängig von der Leistungserbringung an. Es handelt sich für ihn vielmehr um eine grundsätzliche Eigenschaft der Anlage und einen Kennwert, der als Qualitätskriterium definiert ist. Seine Intention zielt also auch auf eine maximale Ausprägung des Verfügbarkeitskennwertes ab, ohne unmittelbar einen Zusammenhang mit der Anlagenleistung zu verfolgen.

Aus den wirtschaftlichen Verpflichtungen der Parteien, also dem Return-On-Invest für den Auftraggeber und dem Liquiditätsrückfluss für den Hersteller, resultiert die Notwendigkeit zu vertraglichen Vereinbarungen über die Leistungserstellung. Darin werden auch Vorgehensweisen und Abnahmekriterien festgelegt [VDI 3649, S. 11], mit denen die Eignung eines Gewerkes für eine bestimmte Funktion geprüft werden soll. Hier ist heutzutage die maßgebliche Funktion des Kennwertes Verfügbarkeit zu sehen. Dabei steht er jedoch in der Regel als isolierter Kennwert und wird nicht direkt in Verbindung gebracht mit der Leistungserfüllung. In Konsequenz folgt, dass beide Parteien trotz der verschiedenen Intentionen an einem hohen Verfügbarkeitskennwert interessiert sind und diesen dementsprechend in einer hohen Ausprägung vereinbaren. Dabei wird weder dem Auftraggeber eine hohe Leistungsermittlung direkt durch den hohen Kennwert garantiert noch kann der Hersteller mit einem leistungsgemäß eingeschränkt funktionsfähigen System eine hohe Qualitätsreputation erzielen. Das resultierende Konfliktpotenzial in dieser Form der Vereinbarung wird jedoch in der Regel nicht als solches erkannt und bewertet. 
An dieser Stelle sollte eine Revision der Verfügbarkeitsbetrachtung für integrierte Materialflusssysteme ansetzen, so dass die beiderseitig induzierte Steigerung geforderter Verfügbarkeitskennwerte durch einen Ansatz substituiert wird, der enger mit der Leistungserbringung des Systems verknüpft ist.

Über diese grundsätzliche Problematik bei der Festlegung der Höhe des Verfügbarkeitskennwertes hinaus mangelt es in der Regel bei den vertraglichen Vereinbarungen auch an einer detaillierten Beschreibung der Abnahmeprozedur und der Berechnung des Verfügbarkeitskennwertes. Hierzu stellen die Richtlinien des VDI und der FEM zwar Anleitungen zur Verfügung, jedoch erfolgt häufig die Übertragung in die vertraglichen Regelungen nur in der Weise, dass ein Bezug dazu hergestellt wird, ohne die genaue Vorgehensweise zu spezifizieren. Damit erfolgt die erforderliche Modellbildung mit den dazu gehörigen Interpretationen erst zu einem Zeitpunkt, wenn eventuelle Konfliktpotenziale in einer Inbetriebnahme bereits offensichtlich geworden sind und die Gestaltung der Abnahmeprozedur von beiden Parteien dem Vorwurf der subjektiven Einflussnahme ausgesetzt ist.

Ein weiteres Element für die Bestimmung der Verfügbarkeit stellt die Beurteilung der auftretenden Störungen dar. Eine grundlegende Auseinandersetzung erfolgt in der VDI-Richtlinie [VDI 4004-4]. Dort sind Störungen auf 4 unterschiedlichen Ebenen unterschieden. Während die theoretische Verfügbarkeit ausschließlich geprägt wird durch technische Ausfälle in Form von Bauteilversagen, berücksichtigt die technische Verfügbarkeit auch Bauteilausfälle mit resultierender Störung auf Grund von Verschleiß. Darüber hinaus existieren noch die operationelle und die praktische Verfügbarkeit. Der erste Kennwert umfasst zusätzlich Störungen, die aus dem System selbst heraus erwachsen oder aus seinem Betrieb resultieren. Die praktische Verfügbarkeit grenzt zusätzlich noch die Störungen ein, die durch Einflüsse von außerhalb des Systems induziert werden. Auch diese Betrachtungsebene verlangt Abstimmung und Festlegung zwischen den Parteien als Grundlage für einen Verfügbarkeitstest [VDI 3649, S. 12; FEM 9.222, S. 5] und bietet dadurch Konfliktpotenzial. Dies kann einerseits darin begründet liegen, dass eine Störung nicht nachvollziehbar ist, wenn z.B. Ursache und Wirkung temporär oder lokal voneinander entfernt liegen. Andererseits entstehen Unsicherheiten auch dadurch, wenn beispielsweise die Anforderungen des Auftraggebers, im idealen Fall dokumentiert in einem Lastenheft, nicht ausreichend spezifiziert oder die Annahmen zur Ausführungsplanung nicht im Pflichtenheft des Herstellers abgedeckt werden. Hinzu kommen noch die Wechselwirkungen im System mit dem eigenen Betriebs- und Bedienverhalte, die häufig bei der Interpretation von Fehlern unbeachtet gelassen werden oder gar nicht in Zusammenhang gebracht werden können.

Ein weiterer Kritikpunkt in Bezug auf die Anwendung aktueller Verfahren zur Bestimmung der Verfügbarkeit ist die Notwendigkeit, festgelegte Durchsatzzahlen [VDI 3649, S. 3] in ihrer maximalen Ausprägung oder einem relativen Anteil davon für die einzelnen Funktionselemente vorgeben zu müssen. Damit vergleichbar fordert die FEM 9.222 für eine abstraktere Abbildung der einzelnen Funktionselemente die Festlegung von Gewichtungsfaktoren, mit denen die Bedeutung des betrachteten Elementes für die Gesamtfunktion ausgedrückt und in der Berechnung der Verfügbarkeit berücksichtigt wird [FEM 9.222, S. 4]. Insbesondere diese Festlegungen, die Eingang finden in den grundsätzlichen Aufbau des Berechnungsmodells für die Verfügbarkeit, können für komplexe Anlagen mit überschneidenden Materialflüssen, Rückflüssen in geschlossenen Kreisläufen oder überschneidenden Arbeitsbereichen nur mit großer Unschärfe festgelegt werden, da die Ausprägung dieser Materialflüsse zeitabhängig und nicht voneinander unabhängig sein muss. Damit ist eine Maximalwertbetrachtung für die Durchsatzkennwerte oder Gewichtungsfaktoren an dieser Stelle ungeeignet. In Folge dessen muss insgesamt angezweifelt werden, dass die Modellierung von abstrakten Blockschaltbildern mit linearen Materialflüssen ein geeigneter Ansatz zur Bestimmung eines Verfügbarkeitskennwertes für Materialflusssysteme mit hoher Komplexität und Varietät ist. Bestärkt wird diese Schlussfolgerung dadurch, dass auch kurzzeitige und lokal begrenzt auftretende Störungen in einem solchen System Auswirkungen auf abhängige Prozesse in der Funktion des Systems auslösen können. In einem solchen Fall kann auch eine geringe Anzahl Störungen, die nicht unmittelbar zu einer massiven Reduktion des Verfügbarkeitskennwertes in der Berechnung führen, trotzdem große Auswirkungen auf die Ausbringung eines Systems bewirken. Wenn jedoch eine derartige Störung, die vom Anwender eines solchen Systems in Form einer reduzierten Leistungsausbringung bemerkt wird, sich nicht in einem Kennwert Verfügbarkeit ausdrückt, muss die Eignung des Verfügbarkeitskennwertes für komplexe Anlagen insgesamt in Frage gestellt werden. Ein Kennwert, der nur auf die Systemabnahme fokussiert, kann an dieser Stelle und insbesondere vor dem Hintergrund der in Abbildung 1 dargestellten wiederholten Umplanung von Materialflusssystemen nicht als sinnvoll angesehen werden. Gleichzeitig ergibt sich hieraus die Anforderung, die Zusammenhänge der Verfügbarkeitsberechnung und der individuellen Festlegung der Einflussgrößen bereits frühzeitig zum Gegenstand von planerischen Prozessen im Zusammenhang mit der Neu- oder Änderungsplanung eines Materialflusssystems zu machen. 


\section{Ansätze zur erweiterten Verfügbarkeitsbetrachtung}

Aus den zuvor dargestellten Zusammenhängen werden die notwendigen Entwicklungen für die Berechnung von Verfügbarkeitskennwerten abgeleitet. Darüber hinaus wird herausgestellt, in welcher Weise das Vorgehen bei der Einforderung von Verfügbarkeitskennwerten, bei der Gestaltung von Materialflusssystemen und beim Nachweis von Verfügbarkeiten angepasst werden müssen, um zu einer effizienteren Nutzung des erweiterten und verdichteten Kennwertes Verfügbarkeit zu gelangen.

Bei der Betrachtung hoch integrierter komplexer intralogistischer Systeme ist die Verfügbarkeit des Gesamtsystems für die Qualität der vom Systembetreiber geforderten logistischen Leistung maßgeblich. Die Gesamtverfügbarkeit bestimmt sich dabei jedoch nicht nur aus der Summe der Verfügbarkeiten der Einzelelemente, sondern wird erst durch die Verknüpfung von elementaren Modulen zu einem komplexen System und der entsprechenden Berücksichtigung der Wechselwirkungen in der Verfügbarkeitsberechnung definiert. Die messbare Verfügbarkeit bestimmt letztendlich die Wirtschaftlichkeit einer Anlage und bildet den Kern der Anforderungen eines Systembetreibers. Daher ist bereits in der frühen Planungsphase bei der Verknüpfung einzelner Module zu dem letztendlich geforderten Gesamtsystem eine Prognose der Verfügbarkeit notwendig, um die Einflüsse einzelner Planungsalternativen auf die Gesamtverfügbarkeit beurteilen zu können. Bereits in dieser frühen Planungsphase (vgl. auch Abbildung 1) der Findung von Materialflussstrukturen sollen Berechnungsverfahren zur Verfügbarkeitsprognose den Planer unterstützen und Kenngrößen für die Bewertung der Planungsalternativen generieren. Dabei sollen bei der Modellierung von komplexen Materialflusssystemen, an die zeitliche Abfolge des Planungsprozesses angepasst und in der Detailtiefe ihrer Betrachtung hierarchisch gegliedert, unterschiedliche Berechnungsverfahren der Verfügbarkeit zur Anwendung gelangen. Ziel eines durchgängigen Ansatzes soll es sein, jeder Stufe der Planung ein geeignetes analytisches Berechnungsverfahren zuzuordnen, wobei zur Berechnung der Verfügbarkeit unterschiedliche Verfahren angewendet werden, die jeweils für spezifische Problemstellungen geeignet sind.

In den frühen Planungsphasen handelt es sich dabei um hoch aggregierte Modelle aus Strukturelementen des Materialflusses, entsprechend der Fokussierung dieser Phase auf die Generierung geeigneter Materialflussstrukturen. Mit der fortlaufenden Detaillierung der Planung und den synchron damit verfügbaren Informationen müssen detailliertere Modelle entstehen. Hier liegt der Fokus der Planungsschritte auf der Auswahl und Dimensionierung der geeigneten technischen Gewerke. Insbesondere hier existiert heute noch eine methodische Lücke, da in diesem kreativen Planungsschritt der Zusammenhang zwischen der Technikauswahl und der verbundenen Rückwirkung auf die Flüsse in den Materialflussstrukturen nicht transparent ist.

Die Bestimmung der Verfügbarkeit eines technischen Systems aus der Verknüpfung der Verfügbarkeitswerte seiner Komponenten auf Basis der elementaren Zuverlässigkeitstheorie, die in der Booleschen Form der elementaren Wahrscheinlichkeitsrechnung und dem Begriff der totalen Wahrscheinlichkeit folgt und vielen Richtlinien zu Grunde liegt, ist für die Betrachtung eines komplexen Materialflusssystems mit einer Vielzahl an unterschiedlichen Komponenten für eine aussagekräftige Bestimmung von Kennwerten besonders komplexer Systeme unzulänglich. In einer frühen Phase der Planung im Rahmen der Systemfindung sind jedoch schnelle und übersichtliche Berechnungsverfahren notwendig, um sofort Auswirkungen von Planungsvariationen beurteilen zu können. In dieser Situation kann die Boolesche Theorie Anwendung finden, um Verfügbarkeiten von einfachen Kombinationen grundlegender Systemelemente, bei denen keine Redundanzen betrachtet werden und die Ausfälle stochastisch unabhängig voneinander sind, zu bestimmen. Bei schärferer Fokussierung auf den endgültigen Systementwurf sollten umfangreichere und leistungsfähigere Methoden zum Einsatz kommen, bei denen zwar ein höherer Aufwand getätigt werden muss, die Kennwerte jedoch präziser und nachhaltiger werden. Diese Stelle in der Kette von Planungsschritten kann die Verfügbarkeitsprognose mit Hilfe der Markov-Theorie einnehmen. Die Berechnung der Verfügbarkeit mit der Markov-Theorie erfolgt über die Betrachtung eines Systems in einem Zustandsänderungsmodell [Bove00]. Gerade komplexe Systeme, die aus mehreren verknüpften Einzelelementen bestehen, können auf diese Weise abgebildet werden. In einem Markov-Modell lassen sich Übergangswahrscheinlichkeiten zwischen Intakt- und Defektzuständen definieren, aus denen die Verfügbarkeit des Gesamtsystems berechnet wird. Mit zeitkontinuierlichen Markov-Ketten kann eine Verfügbarkeitsprognose komplexer Materialflusssysteme abgegeben werden, wobei der Komplexität adaptiver Logistiksysteme in diesem Zusammenhang mit der Verkettung vordefinierter Strukturelemente begegnet werden kann. 


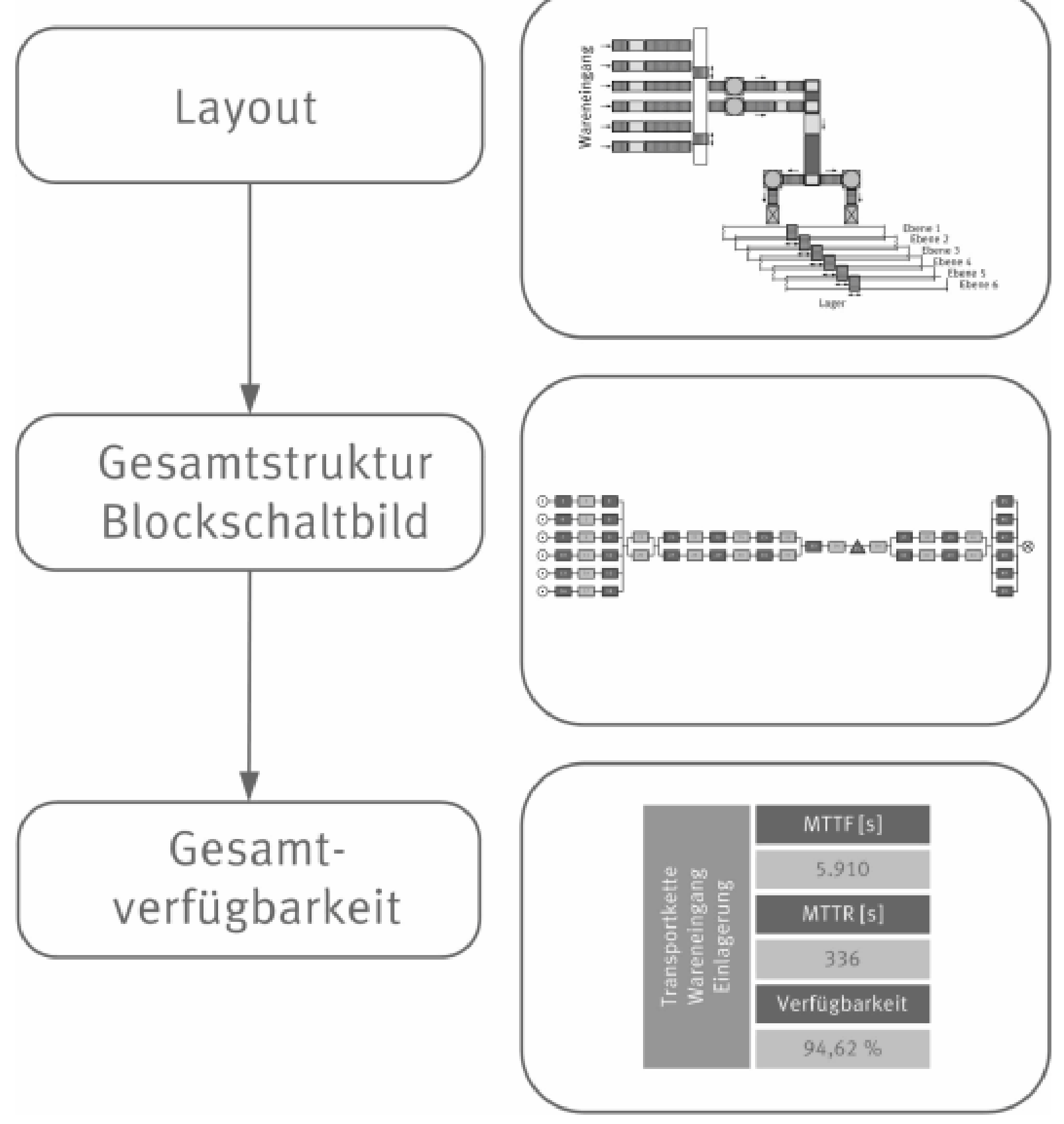

Abbildung 4: Bestimmung der Verfügbarkeit aus der Systemstruktur

Der Übergang vom Einsatz analytischer Methoden zur Verfügbarkeitsberechnung in der frühen Planungsphase der Systemfindung zu den simulativen Verfahren in der Feinplanung komplexer materialflusstechnischer Systeme muss mit adaptierbaren und leistungsfähigen Ansätzen zur mathematischen Modellbildung unterstützt werden. Nur mit erweiterten Verfahren der Modellbildung kann der Übergang von einer kontinuierlichen Beschreibung in eine zeitdiskrete oder ereignisorientierte Umgebung effizient gestaltet werden. So können simulative Ansätze als umfassendste aber auch aufwändigste Methode zur detaillierten und genauen Bestimmung von Verfügbarkeitskennwerten nutzbar gemacht werden und dem Planer belastbare Kennwerte für eine nachhaltige Planung liefern. In diesem Zusammenhang gilt im verstärkten Maße, aber prinzipiell genau so wie für die Verwendung in analytischen Verfahren, dass die Bereitstellung von geeigneten Informationen zur Abbildung des Störverhaltens gewährleistet sein muss. Hierzu muss insbesondere in der Verantwortung von Herstellern und Planern die systematische Sammlung von Ausfall- und Reparaturraten materialflusstechnischer Elemente betrieben werden, so dass Eingangsinformationen für die Anwendung der verschiedenen Verfahren existieren. Die Vorhaltung dieser Daten muss eine statistische Absicherung ihrer Aussagekraft und darüber hinaus eine Anpassung im Hinblick auf Verstärkung oder Reduktion der Kennwerte zum Stör- und Reparaturverhalten umfassen, um Lösungen individueller Anwendungsfälle in Sensitivitätsanalysen zu evaluieren. 
Ein weiterer Ansatz bei der Konsolidierung der analytischen Verfahren zur Berechnung der Verfügbarkeit liegt in der Betrachtung des Reparatur- und Anlaufverhaltens sowie der Integration der daraus resultierenden Einflüsse in die Berechnungsmodelle. Die Motivation dafür liegt in der Betrachtung der Charakteristika komplexer Materialflusssysteme begründet, die eine neue Herangehensweise auf Grund der aus ihrer Komplexität erwachsenden und damit systemimmanenten Dynamik des Anlagenverhaltens erfordern. Kürzer werdende Betriebszeiten eines Gesamtsystems, die Forderung nach Flexibilität und Adaptivität von modernen Materialflusssystemen sowie die systemimmanente Strukturinstabilität erfordern es, die existierenden Berechnungsverfahren zur Verfügbarkeitsprognose neu zu formulieren. Materialflusssysteme können nicht mehr als invariante Systeme über einen längeren Betrachtungszeitraum angesehen werden. Sie passen sich an die Forderungen der Produktionswirtschaft und des Handels nach Flexibilität und Adaptivität an und ändern kontinuierlich ihre Struktur. Somit erfolgt mehrfach innerhalb des Lebenszyklus eines Materialflusssystems die Notwendigkeit der Umplanung und damit einhergehend die wiederholte Generierung und Auswahl von Strukturvarianten respektive der damit verbundenen Aufstellung von Leistungskenngrößen und damit der Bestimmung der Verfügbarkeitskennwerte.

Bei einer detaillierten Betrachtung der Verfügbarkeit von komplexen Systemen sind besonders die Einflüsse des Gesamtsystems auf die einzelne Komponente und wiederum die Reaktion des Systems auf das Verhalten eines einzelnen Bauteils von Interesse, um, auch zur Beurteilung unterschiedlicher Varianten eines Systems, Steuerungsmechanismen für das Materialflusssystem zu entwerfen und mit einer präventiven Instandhaltung schwerwiegenden Verfügbarkeitseinbußen vorzubeugen oder durch geeignete Strategien die Verfügbarkeit gar nicht zu beeinträchtigen. Bei der Verknüpfung grundlegender Strukturelemente bedarf es bei der Berechnung zur Darstellung und Untersuchung eines Materialflusssystems mit seinen Wirkzusammenhängen einer Unterscheidung bezüglich der Lage baugleicher Systemelemente innerhalb des Systems, da die Verfügbarkeiten und Übergangswahrscheinlichkeiten zwischen den Systemzuständen in Abhängigkeit von Anordnung, Einbausituation, Betriebsumgebung und Umwelteinflüssen unterschiedliche Werte annehmen können.

Ziele der vorgestellten Ansätze ist es, dass ein Planer mit Hilfe einer für die Stadien der Planung eines intralogistischen Systems in ihrer Detailtiefe und Granularität hinsichtlich der erfassbaren und für das aktuelle Planungsziel notwendigen Eigenschaften eines Systems konfigurierten Methode, Kennwerte zur Verfügbarkeit und Leistungsfähigkeit einer Anlage vom abstrakten Anlagendesign bis hin zur Komponentenwahl immer detaillierter und verlässlicher ermitteln kann.

\section{5. $\quad$ Fazit}

Zusammenfassend muss festgestellt werden, dass die bestehenden Richtlinien noch keine geeigneten Modelle zur Verfügbarkeitsprognose komplexer Materialflusssysteme bieten, die eine qualifizierte Aussage zulassen. Die Abbildung von charakteristischen materialflusstechnischen Strukturen, wie Redundanzen und Puffer, und charakteristischen materialflusstechnischen Eigenschaften, wie diskrete und zeitpunktgeführte Materialströme, können mit den derzeit existierenden Prognoseverfahren nur unzureichend erfolgen. Ein Berechnungsstandard, der einen exakten Nachweis der Verfügbarkeit für komplexe Materialflusssysteme in der Planungsphase vorschreibt, existiert heute nicht. Damit bleibt der Schluss, dass heutzutage eingesetzte Verfügbarkeitsnachweise auf Grund fehlender Vorschriften und Modelle zur Prognose vielfach auf individuellen Annahmen und Vorgehensweisen beruhen.

Ausgehend von dem untersuchten Verhältnis zwischen Auftraggeber und Hersteller, verbunden mit den analysierten Schwächen über die verfügbaren adaptierten Richtlinien für intralogistische Systeme hin zu den verschiedenen Berechnungsansätzen wurde deutlich, dass sowohl die Notwendigkeit zur intensiveren Einbindung der Verfügbarkeitsbetrachtung in den Planungsprozess von Materialflusssystemen besteht, als auch Potenziale im Spannungsfeld der aktuellen Vorgehensweise und Berechnungsmethoden existieren, die eine Verbesserung der Grundlagen für einen Kennwert Verfügbarkeit versprechen. Dabei gilt es, die methodische Lücke zwischen den einfachen Blockschaltbildbetrachtungen und der detaillierten Modellierung in der Materialflusssimulation mit aufeinander aufbauenden Methoden zu schließen, damit ein effizienter Einsatz in den verschiedenen Phasen des Nutzungszyklus eines Materialflusssystems möglich ist.

Die dargestellten Zusammenhänge zeigen insgesamt die Notwendigkeit, die Abläufe und Methoden bei der Auseinandersetzung mit dem Kennwert Verfügbarkeit von einer funktionsorientierten Herangehensweise weg und hin zu einer verstärkt prozessorientierten Sichtweise zu verändern. 


\section{Literatur}

[Arno05]

[Biro91]

[Bove00]

[Eber91]

[FEM 9.221]

[FEM 9.222]

[Fran93]

[Gaed77]

[Günt02]

[Hörs94]

[Knez93]

[Salz87]

[Schm05]

[VDI 3581]

[VDI 3649]

[VDI 4001-2]

[VDI 4003-1]

[VDI 4004-4]
Arnold, Dieter; Furmans, Kai: Materialfluss in Logistiksystemen; Reihe: VDI-Buch; Berlin, Heidelberg (usw.): Springer, 2005.

Birolini, A.: Qualität und Zuverlässigkeit technischer Systeme. 3. Auflage, Berlin, Heidelberg (usw.): Springer, 1991.

Vom Bovert, E.-M.: Modellerstellung zur Verfügbarkeitsprognose komplexer Förder- und Lagersysteme; Dissertation Universität Dortmund, Dezember 2000.

Eberling, H.: Verfügbarkeit von Materialflußsystemen in der Planungsphase. IN: Fördertechnik 60(1991) 8, S. 54-57.

FEM (Fédération Européenne de la Manutention) 9.221: Leistungsnachweis für Regalbediengeräte, Zuverlässigkeit Verfügbarkeit, 10.1981.

FEM (Fédération Européenne de la Manutention) 9.222: Regeln über die Abnahme und Verfügbarkeit von Anlagen mit Regalbediengeräten und anderen Gewerken, 06.1989.

Franke, W.: Zur Verfügbarkeit von Transport- und Lageranlagen. IN: Fördern und Heben 43(1993)5, S. 295-303.

Gaede, K.-W.: Zuverlässigkeit - mathematische Modelle. München: Hanser, 1977.

Günthner, W.; Heinecker, M.; Wilke, M: Materialflusssysteme für wandelbare Fabrikstrukturen. IN: Industrie Management 18(2002)5, S. 8-10.

Hörstmeier, R.: Fördersysteme mit Kennzahlen bewerten Teil 1. IN: Technica 43(1994)11, S. 21-26.

Knezevic, J.: Reliability, Maintainability and Supportability: A Probabilistic Approach. London: McGraw-Hill, 1993.

Salzer, K. W.: Wie wichtig ist die Verfügbarkeit?, In VDI Berichte 636, Düsseldorf: VDIVerlag, 1987.

Schmidt, T.: Systemtechnische Ansätze zur Erfüllung neuer Anforderungen an Lagersysteme. In: eLogistics journal 2005, ISSN 1860-5923. http://www.elogisticsjournal.de/archiv/2005/3/innovative_lagersysteme/Innovative_Lagersysteme.pdf DOI 10.215/LJ_Not_Ref_d_Schmidt_0320051

VDI-Richtlinie 3581: Zuverlässigkeit und Verfügbarkeit von Transport- und Lageranlagen, April 1983. Berlin: Beuth Verlag, 1983.

VDI-Richtlinie 3649: Anwendung der Verfügbarkeitsrechnung für Förder- und Lagersysteme, Januar 1992. Berlin: Beuth, 1992.

VDI-Richtlinie 4001 Blatt 2: Begriffsbestimmungen zum Gebrauch des VDI-Handbuches Technische Zuverlässigkeit, Juni 1986. Berlin: Beuth 1986.

VDI-Richtlinie 4003 Blatt 1: Anwendung zuverlässigkeitsbezogener Programme, Dezember 1985. Berlin: Beuth, 1985.

VDI-Richtlinie 4004 Blatt 4: Zuverlässigkeitskenngrößen, Verfügbarkeitskenngrößen, Juli 1986. Berlin: Beuth, 1986 . 